\title{
Médicos tradicionales mayas y el uso de plantas medicinales, un conocimiento cultural que continúa vigente en el municipio de Tzucacab, Yucatán, México
}

Recibido: 23/09/2016 · Aceptado: 10/11/2016

Genaro Octavio Can Ortiz

Wilian de Jesús Aguilar Cordero*

Rocío Ruenes Morales

Facultad de Medicina Veterinaria y Zootecnia

Universidad Autónoma de Yucatán

\section{Resumen}

La medicina tradicional se practica desde la época prehispánica, aunque ahora es impactada por la modernización y la globalización, lo que causa una erosión cultural. En este trabajo se revaloran los saberes del quehacer de los médicos mayas tradicionales y la vigencia de su conocimiento. Se definió una muestra de diez médicos mayas tradicionales mediante la técnica de muestreo no probabilístico "bola de nieve", se aplicó un cuestionario y se realizaron entrevistas para conocer la cosmovisión sobre el uso de plantas medicinales, las principales enfermedades que se atienden y las plantas utilizadas para mitigarlas. En los resultados se obtuvo que un número reducido de médicos mayas tradicionales siguen efectuando rituales durante la curación; los padecimientos más comunes son diarrea, vómito y calentura, y la especie más empleada en los remedios es Citrus limonia Osbeck.

Palabras clave: Conocimiento tradicional, cosmovisión, plantas medicinales, transmisión.

*Correo electrónico: acordero@correo.uady.mx 


\title{
Traditional Maya doctors and the use of medicinal plants, cultural knowledge that prevails in the municipality of Tzucacab, Yucatán, México
}

\author{
Genaro Octavio Can Ortiz \\ Wilian de Jesús Aguilar Cordero* \\ Rocío Ruenes Morales \\ Facultad de Medicina Veterinaria y Zootecnia \\ Universidad Autónoma de Yucatán
}

\begin{abstract}
Traditional medicine has been practiced since pre-Hispanic times until our days, although at present it is being impacted by modernization and globalization causing cultural erosion. In this work the work of traditional Maya doctors and the validity of their knowledge is reexamined. A sample of ten traditional Maya doctors was selected in a non-probabilistic technique (snowball method) and questionnaires and interviews were applied to learn about the worldview of the use of medicinal plants, the main diseases that are treated and the plants that are used to mitigate them. The results show that a limited number of traditional doctors continue to perform rituals during the healing, the most common ailments being diarrhea, vomiting and fever and the species most used in the remedies was Citrus limonia Osbeck.
\end{abstract}

KEY worDs: Medicinal plants, traditional knowledge, transmission, worldview.

*E-mail: acordero@correo.uady.mx 


\section{Introducción}

El uso de plantas medicinales en México se remonta a la medicina prehispánica, que se confronta con la medicina española a su llegada en 1521, por lo tanto, la medicina tradicional mexicana actual es resultado del encuentro de dos culturas. Las plantas medicinales para las culturas prehispánicas y sus descendientes, tanto indígenas como mestizos, representan un remedio eficaz para curar diversos males, ya sean físicos, psicosomáticos o mágico-religiosos. Con el arribo de los europeos y sus esclavos africanos, se introduce y enriquece el uso de nuevas plantas, que son incorporadas a los tratamientos médicos por parte de los pueblos establecidos (García, Sierra y Balam, 1996).

Este conocimiento tradicional enriquecido a través del contacto con la cultura occidental fue escasamente sistematizado por los pocos europeos que se interesaron en documentar los conocimientos y costumbres de los pueblos sometidos. Entre las obras que se llevaron a cabo con este propósito están aquellas que tratan en parte o en su totalidad sobre botánica y/o herbolaria medicinal, las cuales se realizaron en diferentes épocas. Respecto a las de la época colonial para México se encuentran Historia de las cosas de la Nueva España (1548), Libellus de Medicinalibus Indorum Herbis (1552) e Historia natural de Nueva España (mediados del siglo xvi). En el caso de la Península de Yucatán destacan la Relación de las cosas de Yucatán (siglo xvI) y las crónicas del Chilam Balam de varios poblados y autores de Yucatán, además de estudios como los de Ricardo Osado el Judío, Benjamín Cuevas, Ralph Roys y Narciso Souza.

No obstante, la escasez de ejemplares y la poca difusión de estas obras provoca que sean consultadas y utilizadas por un reducido número de personas. Es importante reflexionar que los conocimientos sistematizados de la medicina indígena en la actualidad no se están transmitiendo de forma completa a las generaciones de jóvenes dentro de las comunidades rurales, debido a varios factores, entre ellos el auge de la medicina alópata, el acceso al servicio de salud institucional y la pérdida de interés de la gente hacia la medicina indígena, lo cual conlleva una erosión cultural que se refleja en un decremento del uso de las plantas medicinales.

Sin embargo, la medicina indígena sigue siendo una opción para el tratamiento de enfermedades, constituye un elemento de identidad cultural y, por su bajo 
costo y su disponibilidad, es accesible a la población (Yam, Quiñones y Pérez, 1992), además de que contribuye como proveedora de atención primaria de salud en el nivel de la comunidad, según la Organización Mundial de la Salud.

Por otra parte, los médicos tradicionales mayas son, en gran medida, los encargados de mantener determinadas especies dentro sus solares y en las comunidades silvestres, al considerarlas importantes por su empleo medicinal, haciendo un uso racional del recurso y aportando a la conservación ambiental con impacto directo en las comunidades vegetales e indirecto en otros grupos de organismos. Por tanto, en este contexto sociocultural consideramos necesario sistematizar los conocimientos de la medicina indígena. El presente trabajo tiene como objetivo principal conocer la cosmovisión de los médicos mayas tradicionales y sus saberes sobre el uso de las plantas medicinales para el tratamiento y curación de enfermedades en el municipio de Tzucacab, Yucatán, México.

\section{Marco referencial}

\section{Medicina alópata y medicina indígena}

Para la medicina alópata, la enfermedad es originada por agentes biológicos, químicos y físicos, y el restablecimiento de la salud depende de la eliminación de esos agentes para conservar la "normalidad" y/o evitar la muerte del individuo. Por su parte, la medicina indígena, en Mesoamérica, además de considerar que la enfermedad surge a raíz de agentes biológicos y físicos, tiene en cuenta causas sobrenaturales o inmateriales, que provocan un desequilibrio corporal. Entre estos fenómenos se encuentran desestabilizadores como el cambio brusco de temperatura que afecta el equilibrio interno frío-calor del cuerpo (Conti, 1972; Viesca, 1976; García et al., 1996) o la energía corporal fuerte de ciertas personas que actúa en otras de energía débil por acción de la mirada o el contacto físico, por ejemplo.

La medicina indígena es un sistema de salud con su manera particular de diagnosticar y de elegir sus métodos de curación, ya que el conocimiento que envuelve es transmitido de generación en generación y está inmerso en una cosmovisión donde los métodos de sanación involucran la espiritualidad por dos razones: por una parte, tiene como presupuesto que el que cura es el creador y el médico solo es un canal entre este y el paciente y, por otra, que 
tales métodos están diseñados para el espíritu. En este sentido, la medicina indígena tiene el propósito de que el paciente recupere su estado natural de armonía (König, 2011).

Esta medicina presenta tres componentes en su funcionamiento: la cosmovisión, el conocimiento y la práctica, y no se mantienen en campos separados, sino que interactúan en un entramado muy estrecho (Balam Pereira, 1991). Según Toledo, Barrera-Bassols y García-Frapolli (2008), la forma en que los seres humanos se apropian de la naturaleza es a través del complejo kosmoscorpus-praxis (cosmovisión, conocimiento y prácticas). Por ejemplo, en el caso de tratamientos herbarios, las características de las plantas, su ubicación, la parte utilizada y la cantidad recomendada (conocimientos) son la base para su preparación y administración (práctica) que se matiza con elementos culturales propios, simbólicos y religiosos (cosmovisión).

En México, específicamente en la Península de Yucatán, la medicina indígena maya cuenta con diversos practicantes comunitarios, también llamados médicos mayas tradicionales, que son por lo general personas adultas pertenecientes a la comunidad en donde ejercen, a las que el grupo social reconoce como dotados de los conocimientos y habilidades para curar, que diagnostican las enfermedades como parte de una idea de la causalidad que es compartida por el grupo y cuyo sistema de creencias, conceptos y prácticas los distingue claramente de los terapeutas de la medicina alópata (Yam et al., 1992).

Los diversos médicos mayas tradicionales de Yucatán se clasifican, según Yam, Quiñones y Pérez (1992), dependiendo de la actividad que desempeñen, en:

1. Parteras. Son las encargadas de tratar las enfermedades de la mujer y de los recién nacidos, atienden a las mujeres durante el embarazo, el parto y después de este. Generalmente son mujeres, aunque también existe un número reducido de hombres.

2. Sobadores. Restablecen a las personas que sufren golpes, torceduras, dislocaciones y pequeñas luxaciones, utilizando una serie de masajes en el cuerpo del paciente.

3. Hierbateros. Se dedican a tratar las enfermedades a base de plantas y tienen gran conocimiento acerca de estas, de sus propiedades y temperamentos. 
4. H'menoob. Realizan ceremonias agrícolas, poseen conocimientos acerca de las enfermedades y de los recursos para tratarlas. Además curan males de tipo mágico-religioso mediante santiguos, cambios, ceremonias, rezos, etc. Este cargo solo es desempeñado por hombres.

5. Curanderos. Curan males de tipo mágico-religioso por medio de plantas, animales, santiguos y rezos, pero no efectúan ceremonias agrícolas.

Las parteras y los sobadores adquieren sus conocimientos en la práctica, al ser ayudantes de sus papás o de otra persona que les ha querido transmitir sus experiencias. Por otro lado, los h'menoob y los curanderos tienen una forma especial de adquirirlos; se considera que son personas predestinadas para aprender el oficio y se cree que son portadores de un poder divino que les permite curar todo tipo de enfermedades. Los hierbateros pueden adquirir sus conocimientos de una sola de las dos maneras anteriores o de ambas a la vez. Es necesario aclarar que dentro de cada tipo de médico existen especialidades; por ejemplo, entre los hierbateros se pueden encontrar aquellos que se especializan en tratar mordeduras de serpientes, o entre los sobadores están quienes lo hacen en componer dislocaciones, fisuras y fracturas óseas (Yam et al., 1992).

\section{Estudios sobre medicina indígena en Yucatán}

En la Península de Yucatán, los escritos sobre el tema son escasos. El primero en aparecer fue Relación de las cosas de Yucatán, de Fray Diego de Landa, escrito en el siglo xvi, que aunque trataba de diversos temas sobre los mayas de esa época, contiene un apartado que habla de la flora de Yucatán mencionando algunas plantas medicinales.

Los libros del Chilam Balam son fuentes de preservación de los mayas de fines del siglo xvi al siglo xvir que aprendieron a leer y escribir en español de aquellos tiempos y redactaron varios escritos con temas sobre medicina, astrología, religión y mitología, con fuerte relación a sus costumbres y creencias precoloniales, que ahora llevan el nombre del poblado donde fueron realizados o descubiertos. Así tenemos el Chilam Balam de Ixil, Kaua y Nah, que son los que tratan de herbolaria medicinal, pero también se encuentran los de Chumayel, Tizimín, Maní, Tekáx y Tusik (Andrade y Peniche, 1989).

El Ritual de los Bacabes al parecer es originario de Nunkiní, Campeche, de finales del siglo xvi. Ostenta el estilo literario maya con conceptos médicos, 
mágicos y religiosos de ese grupo casi sin influencia europea. Los textos son en su mayoría conjuros para la curación, producto de una combinación de conocimientos médicos, botánicos, mágicos y religiosos, presentada en lenguaje literario y esotérico (García et al., 1996).

La obra de Ricardo Osado -o Giovanni Franceso Mayoli-, de 1750 a 1760, Medicina doméstica, escrita en Valladolid, Yucatán, contiene un gran número de recetas que se remonta hasta el siglo xvi. Como fue difundida en el suroeste por medio de manuscritos, ha ido cambiando y aumentando conforme pasan las generaciones, formando un compendio de la herbolaria medicinal de los siglos xVII y xviII (Lozoya, 1984).

Entre los primeros textos del siglo xx se halla el de Joaquín y Juan Dondé Apuntes sobre las plantas de Yucatán, de 1907, en el cual realizan descripciones botánicas de diversas plantas del estado y su utilidad, principalmente en el campo medicinal, pero también señalan su empleo como utensilios en la vida diaria y usos artesanales. Enseguida está Plantas medicinales de Yucatán y Guía médica práctica doméstica, del doctor Benjamín Cuevas, de 1913, donde redacta descripciones botánicas, propiedades terapéuticas, usos comunes, método y dosis de 211 plantas; además incluye un manual o guía donde describe enfermedades, sus síntomas y su tratamiento mediante recetas con plantas o derivados de ellas y diversas sales, acumulando en total 333 recetas; también agrega ilustraciones de muchas de las plantas que menciona. La obra de Ralph Roys The Ethno-Botany of the Maya, de 1931, describe enfermedades de las comunidades rurales de Yucatán y los remedios para mitigarlas; gran parte de su información estuvo basada en el trabajo de Osado. En 1945 se publica Apuntes relativos a la flora de Yucatán, de Narciso Souza, donde trata una amplia variedad de plantas medicinales, con su clasificación y descripciones. Souza también fue autor de una obra con valor artístico y científico por las plantas allí dibujadas y las recetas que reunió. Sin embargo, no la concluyó, y Mendieta y Del Amo publicaron en 1981 Plantas medicinales de Yucatán, que incluye además de parte de la obra de Souza, una sinonimia popular y científica, parte de las plantas usadas y elaboración de la receta y una bibliografía sobre herbolaria medicinal histórica y contemporánea de la zona (Lozoya, 1984).

Para fines del siglo xx se encuentran diversos trabajos desarrollados por el Centro de Investigación Científica de Yucatán: catálogo de "Plantas medicinales 
de uso común en Yaxcabá, Yucatán”, de Méndez et al. (2003); “Evaluación de la actividad biológica de plantas medicinales nativas de la Península de Yucatán”, de Vera (2004), y "Estudio de Tridax procumbens L. para obtención de metabolitos con actividad antiprotozoaria”, de Martín (2009); y del Centro de Investigación y de Estudios Avanzados Unida Mérida como los realizados por el doctor Gilberto Balam, entre los que destaca su libro Flora maya medicinal (2003).

\section{Materiales y métodos}

\section{Descripción del área de estudio}

El municipio de Tzucacab está situado al sur del estado de Yucatán, a unos $135 \mathrm{~km}$ de la ciudad de Mérida. Incluye 13 comisarías: Blanca Flor, Catmis, Caxaytuk, Corral, Dzi, Escondido, Ek Balam, Noh Bec, Piste Akal, Polhuacxil, Sacbecan, Tigre Grande e Xcobiacal. Colinda al norte con los municipios de Tixméuac y Chacsinkín, al sur con el municipio José María Morelos del estado de Quintana Roo, al este con Peto y al oeste con Tekáx (figura 1).

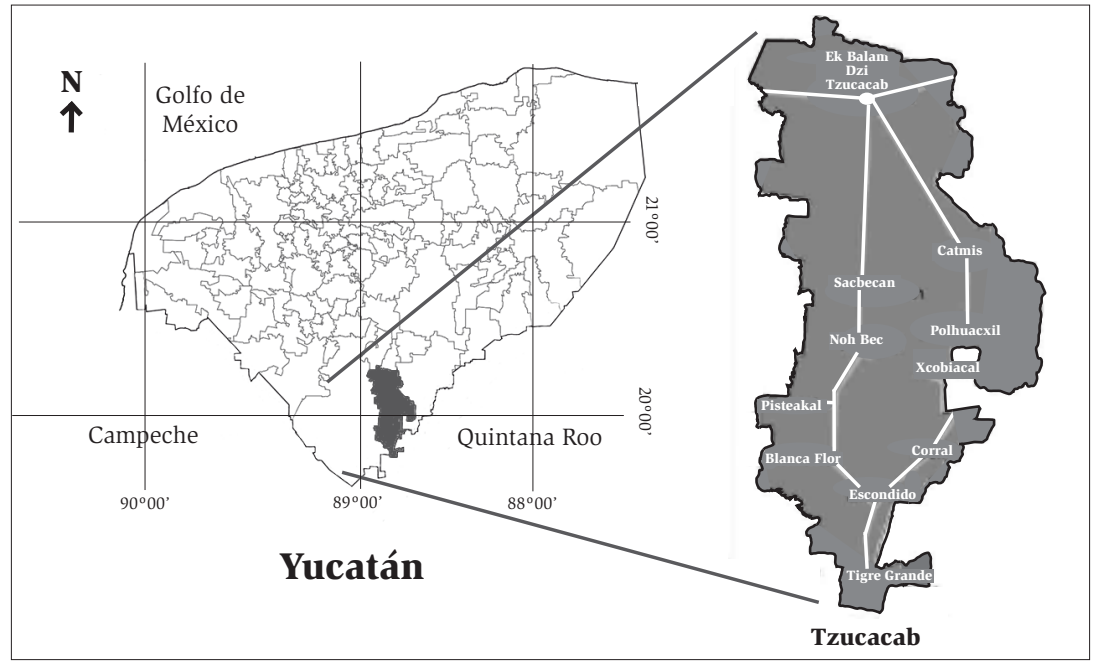

Fuente: Modificado de May (2007). 


\section{Clima}

El municipio presenta un clima cálido-húmedo con régimen de lluvias en verano. El promedio de temperatura es de $26.4{ }^{\circ} \mathrm{C}$, la precipitación total anual, de 1000 mm (Zamora, 2007). Las vegetación está catalogada como selva mediana subcaducifolia y el paisaje se ha fragmentado por la agricultura, la ganadería y la explotación forestal (Flores y Espejel, 1994; Zamora, 2007), lo cual ha generado un gran porcentaje de selvas secundarias.

\section{Datos demográficos}

De acuerdo con datos del Instituto Nacional de Estadística y Geografía (INEGI, 2007), la población total es de 13564 individuos, con 6900 hombres y 6664 mujeres. El total de hogares es de 2974 , y el tipo dominante de hogar es el nuclear, donde los padres viven solo con los hijos solteros, con un promedio de cuatro integrantes en cada hogar. Asimismo, $94.86 \%$ de los habitantes mayores de 5 años es bilingüe y $5.14 \%$ habla únicamente maya.

\section{Servicio de salud}

De la población total, solo 14.68 \% es derechohabiente de alguna institución, la mayoría del Instituto Mexicano del Seguro Social (IMss) (1503 personas), y 531 personas del Instituto de Seguridad y Servicios Sociales de los Trabajadores del Estado (ISSSTE) (INEGI, 2007). La cabecera municipal cuenta con instalaciones del Imss, de Servicios de Salud de Yucatán (ssy) y del Sistema Nacional para el Desarrollo Integral de la Familia (DIF). Casi todas las comisarías tienen instalaciones de ssy, excepto en los poblados de Escondido y Tigre Grande, que no cuentan con ninguna instalación de salud.

\section{Educación}

De la población mayor de 6 años, 80.1 \% (9 342 habitantes) sabe leer y escribir, y 19.9 \% es analfabeta (2 317 habitantes) (INEGI, 2007). La cabecera municipal cuenta con preescolar, primarias, secundarias y bachillerato, mientras que en el resto del municipio hay preescolar y primarias, aunque estas últimas en algunas comisarías en calidad de teleprimaria dirigida por un solo maestro, como en los pueblos de Escondido y Tigre Grande. 


\section{Actividades productivas}

Las principales actividades productivas desarrolladas de forma tradicional en el municipio de Tzucacab son la agricultura con maíz, sandia, camote y otros; en el solar se pueden encontrar bovinos, porcinos y ovinos, la avicultura y la apicultura (INEGI, 2007), aunque también son comunes los cultivos de frutales, hortalizas, plantas medicinales y de ornato.

El municipio posee 647 ha de tierras mecanizadas en donde se cultiva maíz, papaya, melón, calabaza, chile y sandía. Para el municipio destaca la ganadería con 42331 bovinos y 4168 porcinos, la apicultura con 1580 colmenas de abeja europea, y las artesanías, por ejemplo el urdido de hamacas y las prendas de vestir como los ternos e hipiles. En la selva se lleva a cabo la extracción forestal de productos maderables y no maderables como la recolección de hojas de palma y la cacería (Segovia, 2001).

\section{Metodología}

Un paso fundamental fue un primer acercamiento con las autoridades locales en todas las comisarías del municipio de Tzucacab para la identificación de los médicos mayas tradicionales. Se empleó la técnica de muestreo no probabilístico denominado "bola de nieve", que consistió en que, por medio de los comisarios ejidales, se identificaran algunos médicos tradicionales, a los que después se contactó para explicarles la naturaleza del estudio y con su ayuda ubicar a otros médicos tradicionales mayas (Babbie, 1999). Esta etapa se realizó durante octubre de 2008.

Se logró identificar a diez médicos mayas tradicionales, a quienes se les aplicó cuestionario y entrevista. Cabe destacar que, a través de estas dos técnicas de investigación social, se cubrieron los aspectos cuantitativos y cualitativos (Aguilar Cordero, 2000).

\section{Entrevista y cuestionario}

Las entrevistas se efectuaron de noviembre de 2008 a mayo de 2009, y versaron sobre la vigencia de la medicina indígena y sus problemáticas. A través de ellas se conocieron los padecimientos que son atendidos, las plantas para tratarlos 
y la cosmovisión asociada, incluyendo la forma de procesar y administrar, así como la manera de obtener el recurso medicinal.

Para el cruce de técnicas y buscar la confiabilidad y validez de la información se diseñó y aplicó un cuestionario, con la finalidad de obtener información cuantitativa para conocer las principales enfermedades y sus tratamientos con medicamentos tradicionales. De forma paralela a las entrevistas se recolectó material vegetal para su propagación e identificación botánica y se hizo un registro fotográfico de las muestras. El material botánico se depositó en el Herbario Alfredo Barrera Marín de la Universidad Autónoma de Yucatán.

\section{Análisis}

Las entrevistas a los médicos tradicionales mayas fueron grabadas y se transcribieron para su análisis, clasificación y sistematización. Además, para todas las especies se calculó el valor de uso (vu) con la siguiente fórmula: vu $=\left(\sum U \mathrm{Ui}\right) / \mathrm{n}$; donde $\sum$ Ui es la suma de todos los usos mencionados por todos los informantes para una especie, y n es el número total de informantes.

\section{Resultados y discusión}

En el municipio de Tzucacab se identificaron diez médicos tradicionales en tres localidades: uno en Tigre Grande, tres en Noh Bec y seis en la cabecera municipal, $60 \%$ mujeres y $40 \%$ hombres y cuyo rango de edad fue de 43-71 años; el tiempo que han estado practicando la medicina tradicional es de 3 a 55 años, con un promedio de 36.2 años. De los hombres, $30 \%$ tiene como oficio principal ser campesino y $10 \%$ campesino y hierbatero; de las mujeres, $50 \%$ se dedica a labores domésticas y $10 \%$ a la artesanía. La persona cuyo oficio principal es ser hierbatero tiene un pequeño vivero donde cultiva y vende plantas medicinales, aunque cabe mencionar que todos tienen en su casa esta clase de plantas, aunque solo sea para su consumo y no para vender. Los diez médicos tradicionales poseen la especialidad de hierbateros, y tres de ellos cuentan con otra especialidad: dos son sobadores y uno sobador de cirro (atienden la enfermedad, como el tip'te tuch, "latido umbilical", cirro o "cirro ladeado"). Doña Felipa Cauich y doña Marta Castañeda, madre e hija, respectivamente (cuadro 1), manifestaron que durante las curaciones utilizan oraciones e invocaciones a varios ángeles y santos para ayudar en el alivio del padecimiento. 
Médicos tradicionales mayas y el uso de plantas medicinales, un conocimiento cultural que continúa vigente en el municipio de Tzucacab, Yucatán, México

CUADRo 1. Datos generales sobre los médicos tradicionales mayas contactados en el municipio de Tzucacab, Yucatán

\begin{tabular}{|c|c|c|c|c|c|c|c|c|}
\hline & $\begin{array}{c}\text { Médico } \\
\text { tradicional }\end{array}$ & $\begin{array}{c}\text { Oficio } \\
\text { principal }\end{array}$ & Comisaría & $\begin{array}{l}\text { Edad } \\
\text { (años) }\end{array}$ & Sexo & $\begin{array}{l}\text { Años } \\
\text { que } \\
\text { practica }\end{array}$ & $\begin{array}{l}\text { Persona } \\
\text { de quien } \\
\text { aprendió }\end{array}$ & Grado \\
\hline 1 & $\begin{array}{l}\text { Akil, } \\
\text { Valerio }\end{array}$ & $\begin{array}{l}\text { Campesino } \\
\text { y hierbatero }\end{array}$ & Cabecera & 70 & $\mathrm{H}$ & 40 & Abuelos & $\begin{array}{l}\text { Hierbatero } \\
\text { y sobador }\end{array}$ \\
\hline 2 & $\begin{array}{l}\text { Cano, } \\
\text { Miguel }\end{array}$ & $\begin{array}{l}\text { Albañil y } \\
\text { campesino }\end{array}$ & Cabecera & 45 & $\mathrm{H}$ & 22 & $\begin{array}{l}\text { Tío de su } \\
\text { esposa, } \\
\text { suegra y } \\
\text { vecinos }\end{array}$ & Hierbatero \\
\hline 3 & $\begin{array}{l}\text { Castañeda, } \\
\text { Marta }\end{array}$ & Artesana & Cabecera & 43 & M & 24 & $\begin{array}{l}\text { Tío, madre } \\
\text { y otros } \\
\text { médicos } \\
\text { tradicionales }\end{array}$ & Hierbatera \\
\hline 4 & $\begin{array}{l}\text { Catzin, } \\
\text { Mariano }\end{array}$ & $\begin{array}{l}\text { Campesino } \\
\text { y comisario } \\
\text { ejidal }\end{array}$ & $\begin{array}{l}\text { Tigre } \\
\text { Grande }\end{array}$ & 45 & $\mathrm{H}$ & 35 & Padre & $\begin{array}{l}\text { Hierbatero } \\
\text { y sobador }\end{array}$ \\
\hline 5 & $\begin{array}{l}\text { Cauich, } \\
\text { Felipa }\end{array}$ & $\begin{array}{l}\text { Ama de } \\
\text { casa }\end{array}$ & Cabecera & 68 & M & 51 & $\begin{array}{l}\text { Tío-abuelo y } \\
\text { otro médico } \\
\text { tradicional }\end{array}$ & Hierbatera \\
\hline 6 & $\begin{array}{l}\text { Chan, } \\
\text { Edilberto }\end{array}$ & $\begin{array}{l}\text { Albañil y } \\
\text { campesino }\end{array}$ & Noh Bec & 43 & $\mathrm{H}$ & 3 & $\begin{array}{l}\text { Madre, } \\
\text { vecinos y } \\
\text { compañeros } \\
\text { de trabajo }\end{array}$ & Hierbatero \\
\hline 7 & Días, Alicia & $\begin{array}{l}\text { Ama de } \\
\text { casa }\end{array}$ & Cabecera & 67 & M & 55 & De nadie & $\begin{array}{l}\text { Hierbatera } \\
\text { y sobador }\end{array}$ \\
\hline 8 & $\begin{array}{l}\text { Pacheco, } \\
\text { Amira }\end{array}$ & $\begin{array}{l}\text { Ama de } \\
\text { casa }\end{array}$ & Cabecera & 65 & M & 53 & $\begin{array}{l}\text { Madre } \\
\text { y otros } \\
\text { médicos } \\
\text { tradicionales }\end{array}$ & Hierbatera \\
\hline 9 & $\begin{array}{l}\text { Serralta, } \\
\text { Ignacia }\end{array}$ & $\begin{array}{l}\text { Ama de } \\
\text { casa }\end{array}$ & Noh Bec & 68 & M & 42 & Madre & Hierbatera \\
\hline 10 & Uc, Justina & $\begin{array}{l}\text { Ama de } \\
\text { casa }\end{array}$ & Noh Bec & 71 & M & 37 & $\begin{array}{l}\text { Madre y } \\
\text { vecinos }\end{array}$ & Hierbatera \\
\hline
\end{tabular}

Fuente: Elaboración propia (2009). 
Solo uno de los $(\mathrm{n}=10)$ entrevistados cuenta con tres años de experiencia en ser médico tradicional maya; no obstante hace poco tiempo se inició en las prácticas curativas, tiene un conocimiento sobre el tema por encima de una persona promedio de su comunidad, aunque hay dos miembros más de ella que hace algún tiempo se dedicaron a esta labor.

La construcción de las instalaciones de ssy en la comunidad fue un factor que contribuyó a la disminución de los pacientes que atendían los médicos tradicionales mayas, porque aquellos comenzaron a acudir al centro de salud, y a pesar de que la afluencia de gente se concentró en ssy, la consulta, aunque fuera mínima, se mantuvo con los hierbateros. Algo similar sucedió en el área del Puuc (nombre maya-yucateco, significa serranía y se encuentra al sur del estado de Yucatán y al norte del estado de Campeche), ya que al establecerse la medicina institucional (alópata) los servicios solicitados a los hierbateros disminuyeron, pero no desaparecieron por completo (Güemez, 1992 y Gubler, 1996).

Plantas medicinales y enfermedades atendidas por los médicos tradicionales

\section{ESPECIES MÁS UTILIZADAS}

La mayoría de las 142 especies vegetales mencionadas por los hierbateros como medicinales son nativas (77.31\%), muy similar a lo reportado por otros estudios en Yucatán (Narváez, 2001; Polanco, 2004) en cuanto al porcentaje. Esto es de esperarse, ya que son comunidades que han interactuado con las especies nativas durante varios siglos, además de que los poblados están cerca del monte, en el caso de la cabecera, y en cuanto a las comisarías de Noh Bec y Tigre Grande, estas se hallan inmersas en la selva y la accesibilidad de los recursos curativos está al alcance. Asimismo, todas las personas entrevistadas cuentan con plantas medicinales en sus solares, en donde se encuentra el mayor porcentaje de las especies introducidas (22.69\%), en particular las especies de las familias Lamiaceae, Rutaceae, Liliaceae y Crassulaceae, pues, como mencionaron varios hierbateros, "es mejor tenerlas al alcance porque puede haber ocasiones que no tengas tiempo de ir a buscarlas [al monte]".

La especie más utilizada para los remedios es el limón (Citrus limonia), conocido también como limón indio o limón "pais”; después están el zapote o ya' (Manilkara zapota), el k'anan (Hamelia patens), la naranja agria o paakal (Citrus aurantium), la menta (Mentha citrata), el poleo (Micromeria brownei) 
y el kakaltun (Ocimum campechianum); la familia botánica mejor representada es la Lamiaceae, divergiendo con los trabajos de los autores anteriores, que reportaron la Fabaceae (leguminosas) como la mejor representada. En el presente estudio, esta familia botánica ocupó el cuarto lugar junto con la Rutaceae.

\section{VALOR DE USO}

Las especies con mayor vu fueron el limón (C. limonia) y el "cunde amor" o yakunaj aak' ( $M$. charantia), que son empleadas en seis padecimientos, exhibiendo una importancia relativa en comparación con otras especies en la misma comunidad, por tanto, dentro del mismo contexto cultural (cuadro 2). Al respecto se menciona que un alto número de citaciones para un uso específico de alguna planta señala una mayor probabilidad de eficacia real sobre enfermedades (Bermúdez y Velásquez, 2002). En estudios enfocados a la investigación de sustancias activas contra padecimientos o enfermedades, los vu muestran especies con un elevado potencial de actividad, sugiriendo plantas con una fuerte eficacia farmacológica en la región. En este estudio solo nos indicó que las especies con altos coeficientes de vu son muy apreciadas por los médicos tradicionales mayas, quienes creen muy considerablemente en sus propiedades curativas.

Cuadro 2. Especies con mayor uso

\begin{tabular}{|c|c|c|c|c|c|}
\hline Especie & $\mathrm{vU}$ & Especie & vU & Especie & vU \\
\hline Citrus limonia & 0.6 & Momordica charantia & 0.6 & Citrus auratium & 0.5 \\
\hline Croton flavens & 0.5 & Croton reflexiflius & 0.5 & Manikara zapota & 0.5 \\
\hline Micromeria brownei & 0.5 & Persea americana & 0.5 & Hamelia patens & 0.4 \\
\hline Ipomea heterodoxa & 0.4 & Malmea depressa & 0.4 & Mentha citrata & 0.4 \\
\hline $\begin{array}{l}\text { Ocimun } \\
\text { campechianum }\end{array}$ & 0.4 & Salvia micrantha & 0.4 & Urechites andreuxii & 0.4 \\
\hline Anís en grano & 0.3 & $\begin{array}{l}\text { Cinnamomun } \\
\text { zeylanicum }\end{array}$ & 0.3 & Citrus sinensis & 0.3 \\
\hline $\begin{array}{l}\text { Malvaviscus } \\
\text { arboreus }\end{array}$ & 0.3 & Mentha sativa & 0.3 & Murraya paniculata & 0.3 \\
\hline Petiveria alliacea & 0.3 & Pluchea symphytifolia & 0.3 & Stenandrium nanum & 0.3 \\
\hline $\begin{array}{l}\text { Tynanthus } \\
\text { guatemalensis }\end{array}$ & 0.3 & & & & \\
\hline
\end{tabular}

Fuente: Elaboración propia (2009). 


\section{Padecimientos y ENFERMEDADES COMUnes}

Los hierbateros atendían en conjunto un total de 81 padecimientos, entre los cuales existía un común denominador para casi todos los médicos tradicionales mayasen en cuanto a frecuencia de consulta: diarrea, vómito y calentura, que son males usuales para zonas rurales de México (Hernández, 2000) y padecimientos comunes en Yucatán (Balam Pereira, 1991). No obstante, estos padecimientos estaban vinculados con enfermedades complejas como disenterías, y las relacionadas con los síndromes o enfermedades culturales como pasmo (estado corporal que tiene diversos síntomas y provoca diferentes padecimientos dependiendo de los órganos o zonas en que se ubique, de modo que se puede tener escalofríos, comezón, calentura, dolor, diarrea, entre otros, pero cuando una persona se encuentra en estado de pasmo o pasmado, por lo general presenta sudor de noche, su cuerpo está pegajoso y tiene un olor desagradable) y mal de ojo (resulta del contacto entre una persona de mirada fuerte y un niño al cual enferma solo con verlo), entre otras; estos médicos no se remiten simplemente a atender el padecimiento, sino a comprenderlo lo mejor posible para luego tratarlo, ya sea causado por factores materiales o mágico-religiosos.

Los tres padecimientos tratados con más frecuencia concuerdan con los que más atiende ssy de la cabecera de Tzucacab, según su director Nicolás López (comunicación personal). Esto permite constatar que la población consulta a los médicos alópatas y a los tradicionales por las mismas razones, aunque también depende de factores como el recurso monetario disponible, la confianza, la cercanía al consultorio o al médico tradicional, o la decisión fundamentada en el sistema de creencias culturales. Esta última opción está vinculada con las condiciones ambientales-culturales-lingüísticas que imperan en las comunidades (Ku, 1992; Gubler, 1996). En este sentido, los médicos tradicionales mayas hicieron referencia a que ciertos padecimientos como el pasmo y el mal de ojo "son enfermedades que no son de doctor", entendiendo que estos males no pueden ser curados por un médico alópata, porque no cuenta con los recursos y/o porque no tiene la misma cosmovisión. Por tanto, solo conociendo los valores reales o imaginarios de una comunidad o sector de la población es como podremos entender los aspectos lógicos del proceder de sus integrantes para el logro de la salud (Lagarriga, 2000). 
La herencia cultural: aprendiendo y transmitiendo los conocimientos médicos tradicionales

De los médicos mayas tradicionales, 80 \% aprendió como principal fuente de un familiar, similar a lo reportado anteriormente (Gubler, 1996; Polanco, 2004). El señor Chan, hierbatero de Noh Bec, se acercó definitivamente a estas prácticas debido a que sufrió de piedra y arenilla (litiasis renal y en la vejiga urinaria, respectivamente), y al no tener recursos económicos para la operación buscó consejos con sus compañeros de trabajo para tratar su padecimiento, probando varias recetas antes de obtener resultados positivos con la medicina indígena maya.

La señora Cauich y la señora Castañeda, madre e hija, viajan constantemente a Tahdziú, poblado al norte de Tzucacab, Yucatán, donde han aprendido de otro médico tradicional -aunque la señora Castañeda inició su aprendizaje con una hierbatera de su comunidad con la que no compartía parentesco.

Otra forma de aprendizaje más enfocado a la parte mística fue la comentada por la señora Días:

Yo soy naturalista de nacimiento, desde la edad de 9 años empecé a tener esa corporación [obtener conocimientos curativos de ciertas plantas a través de una fuente sobrenatural], pero pues no lo creían nadie, ya después comencé a desarrollar [las prácticas médicas]. Cuando iba yo a jugar con los niños, andaba recogiendo las hierbas [curativas], hasta hay muchas personas que me tiraban a la loca, [y decían] "esa chiquita solo está jugando, vacilando". Entonces se dieron cuenta que todas las hierbas que recogía, pues sí me daban resultados [curaban] [entrevista personal, 2009].

\section{Disminución del uso de la medicina indígena}

Los hierbateros consideraron que la medicina indígena y el uso de plantas medicinales está disminuyendo por tres razones: 1) aumento de las instituciones de salud (ssy, Imss, Similares, etc.), 2) pérdida de creencia en los tratamientos y recursos tradicionales y 3) uso de agentes químicos. Un ejemplo de esta realidad social es que varias personas de las comunidades, sobre todo las de Tigre Grande, buscan los servicios de medicina alópata acudiendo a la comisaría de Corral o a la cabecera municipal, lo cual es notado por los hierbateros, como 
mencionó el señor Catzin:

[Ahora] es más fácil ir con el doctor [médico alópata], si hasta los parteros dicen que es más fácil ir con el doctor, aun si lo pueden atender mandan a las parturientas al hospital porque es más fácil. Mientras llegas al hospital, inyectan a la mujer y forzan al bebé para que nazca, después la mujer sufre mucho, no es como con el partero que sabe cómo hacerle para que la madre no sufra bastante. Sí hay hierbateros y parteras, pero todo se está perdiendo, ahora ya es diferente la situación [entrevista personal, 2009].

Aunque también se indicó que la disminución de estas prácticas no es un suceso total:

Solo en algunas zonas [está desapareciendo], por ejemplo en las ciudades, porque ya no hay las plantas y la gente ya no creen en ellas. Pero en los pueblos como este, no, porque todavía existen las plantas medicinales y hay la gente que cree y sana con ello [Castañeda, entrevista personal, 2009].

Para evitar la pérdida del conocimiento cultural sobre el uso y aprovechamiento de las plantas medicinales y de la medicina indígena, tres médicos mayas tradicionales proponen:

1. Invitar a la gente de los ejidos para que no dejen de utilizar la medicina indígena y que el gobierno estatal apoye a quienes deseen usar este conocimiento cultural.

2. Ir a las escuelas y preguntar a los jóvenes quienes son los que están interesados en aprender sobre la medicina indígena y seleccionarlos para enseñarles a escoger las plantas medicinales, preparar las medicinas y los tratamientos que hay que hacer para las diversas enfermedades.

3. Enseñar en las escuelas el uso de las plantas medicinales, así como dicen que se enseñan las clases de lengua maya (Chan, Castañeda y Cauich, entrevista personal, 2009).

La transmisión del conocimiento cultural de la medicina indígena maya es de persona a persona, sobre todo en la familia, principalmente a sus hijos de forma activa (50\%). Por otro lado, algunos hierbateros no demostraron tener intenciones abiertas y activas en trasmitir sus conocimientos a algunos de sus 
familiares o algún otro conocido, caso en el que se encuentra la otra mitad de los entrevistados; no obstante, de este $50 \%, 40 \%$ transmite sus conocimientos a quien lo solicite, sean sus hijos, los vecinos o las personas interesadas que van a consultar. A pesar de esto, la señora Castañeda comentó que cuando le preguntan sobre cómo se prepara y administra algún medicamento, no responde porque la persona pudiera intentar hacerlo y cometer errores, poniendo en riesgo a otro individuo, la cual es una actitud sensata, pues la persona que administre medicamentos naturales de forma errónea puede causar daño y provocar la desvalorización de la medicina indígena por considerarla ineficiente, amén del daño físico y emocional ocasionado.

A pesar de la compleja situación de la medicina indígena maya frente a la alópata, la primera aún es una opción viable para las personas de las comunidades rurales, como se constató en la presente investigación; además, la medicina indígena maya posee áreas de notable eficacia y resulta económicamente más factible para una gran parte de las poblaciones indígenas y rurales (Yam et al., 1992; Anzures, 2000). La medicina indígena maya se ha mantenido porque responde a formas culturales y necesidades de grupos mayoritarios; es por ese motivo que, en opinión de la señora Castañeda, la creencia en las plantas medicinales se conserva más en las comunidades, dado que existe el recurso, y existe un lazo cultural importante: la cosmovisión que comparten sus miembros.

\section{Cosmovisión de los médicos tradicionales}

Cuando un paciente llega a consultar a un médico maya tradicional, lo primero que este hace es el diagnóstico, el cual consiste en una comunión entre el médico tradicional y el paciente, identificando sus síntomas. Este rito terapéutico es un mecanismo de disolución de diferencias, se desvanece el binomio curandero-paciente para transformarse en un monomio de identidad recíproca, médico y paciente se ven como uno solo a pesar de ser distintos (Balam Pereira, 1991). El diagnóstico puede dividirse en dos grupos: por signos y síntomas, y por adivinación (García et al., 1996). Todos los médicos manifestaron utilizar el diagnóstico de la enfermedad por los signos y síntomas que presenta el individuo, aunque también señalaron (20\%) que realizan un ritual religioso (sincrético) antes de iniciar el diagnóstico y durante este, que consiste en: 
Primero debes tener un lugar donde estén todas las imágenes, la de Dios [Jesucristo] porque él hizo todo el universo y la tierra, después los cuatro ángeles, Gabriel, Miguel, Rafael y Uriel, puede ser de figuras o en cuadros. Yo comencé a trabajar con estos, San Martín Caballero, que es el dios de los pobres, también a la Virgen de Guadalupe y al Niño de Atocha [Castañeda, entrevista personal, 2009].

El altar es el espacio físico usado por los algunos médicos mayas tradicionales para efectuar su primer diagnóstico para la remediación y/o curación de las personas. Este sitio es considerado la mesa de trabajo, donde se encuentran las imágenes o láminas de santos, cruces, velas y flores (Gubler, 1996). Sin embargo, no todos tienen estos altares, porque no todos emplean oraciones e invocaciones al momento de curar; 80 \% diagnostica a través de los síntomas y administra los medicamentos.

El $20 \%$ restante integra elementos religiosos durante el diagnóstico, sin ser por eso un diagnóstico por adivinación, realizando el siguiente ritual:

[Primero], cuando sean niños, vas a invocar a la Virgen de Guadalupe, que es la madre de todo, y al Niño de Atocha, porque es un infante, con ellos vas a pedir por la salud del niño. No se maneja nada de suerte, solamente lo tocas y miras, le tocas su frente, su espalda, las manos, su cuerpo y tomas su pulso, si notas que está caliente vas a pedir: "Oh, nuestra madre santísima de Guadalupe, yo te pido este fervor y favor te pido, por... (vas a decir el nombre del niño), que tú apartes de la enfermedad de este niño", en este momento vas a utilizar la ruda o sip'che'. Con unos gajos empiezas a invocar a la santísima Virgen de Guadalupe y rezas Dios te salve, Ave María y un salve, mientras santiguas al niño y lo vas bajando con los gajos hasta el suelo y después los tiras por detrás del niño. Después remojas con alcohol otro gajo nuevo y bañas al niño y se termina [Castañeda, entrevista personal, 2009].

La curación se efectúa mediante la invocación de diversos ángeles y santos, y con el ramo de ruda (Ruta chalapensis) o sip'che' (Bunchosia swartziana) según con el que se cuente, porque para este fin son equivalentes- se comienza a eliminar de forma simbólica el mal que afecta a la persona. Se pasa por el cuerpo el ramo de arriba hacia abajo en un acción denominada limpia, barrido o escobillado, y se realiza previo a la administración de las plantas para facilitar su efectividad (Lagarriga, 2000), lo cual también fue señalado por la señora Castañeda. 
Balam Pereira (1991) apunta que el curandero maya encuentra en la religión la explicación a su eficiencia médica; lo divino se expresa a través de la planta, de la mente y de la oración. Por ello, la medicina indígena maya es un elemento de identidad cultural, en donde los participantes (médicos mayas tradicionales y pacientes) se comprenden mutuamente (Yam et al., 1992). Además, el paciente que borra de sí la tensión de su rostro y las acciones del h'men en su terapia diagonal aprehende lo humano, lo natural y lo divino. Ambos tienen la misma escala de valores, y es el médico maya quien los maneja para restaurar la salud o, al menos, reducir la tensión y el malestar del paciente (Balam Pereira, 1991).

\section{Conclusiones}

La especie más empleada por los médicos tradicionales mayas en distintos tratamientos fue el limón (C. limonia), que registró más usos en diversas recetas; también es una especie de uso común, ya que se utiliza en combinación con otras plantas para tratar la calentura. Otras especies de uso común fueron zapote ( $M$. sapota), k'anan (H. patens), naranja agria (C. aurantium), menta (M. citrata), poleo (M. brownei) y kakaltun (O. campechianum), empleadas en combinación con algunas más y en diferentes proporciones para tratar calentura, diarrea y vómito, padecimientos muy habituales en el municipio, tanto para los centros de medicina alópata como para los médicos tradicionales.

La transmisión oral es la fuente primordial de enseñanza de la medicina indígena maya, sea de forma activa o pasiva, es decir, que el médico tenga intención deliberada de enseñar (familiares principalmente), o que por curiosidad de otra persona este le transmita sus conocimientos (familiares y vecinos).

Un bajo porcentaje de los médicos mayas tradicionales (20\%) utiliza la invocación de ángeles y santos, usando un lugar especial con imágenes, oraciones y movimientos específicos para remediar los padecimientos. No obstante, este método no es un diagnóstico ni una curación a través de la adivinación o suerte, como indicó alguno de los médicos tradicionales. Constituye simplemente una integración religiosa en relación con la curación, que viene enriquecida con el empleo de plantas, objetos u otro elemento material.

La medicina indígena maya continúa siendo una opción primaria de atención de la salud en las comunidades estudiadas, y aun con sus altibajos en su transmisión, existe cierto nivel de apreciación e interés por este conocimiento. 


\section{Fuentes consultadas}

Aguilar Cordero, W. (2000). Relevancia de los aspectos socioculturales en la conservación y el manejo de la vida silvestre. En O. Sánchez, C. Donovarros y J. Sosa (eds.), Conservación y manejo de vertebrados en el trópico de México: Diplomado en Conservación, Manejo y Aprovechamiento de Vida Silvestre. Unidos para la Conservación/Agrupación Sierra.

Andrade Canul, O. E. y Peniche Zapata, H. S. (1989). Plantas medicinales a través del Chilam Balam Nah de Teabo y su estudio comparativo. Tesis de licenciatura. Mérida, Yucatán: Facultad de Ciencias Antropológicas. Universidad Autónoma de Yucatán.

Anzures, M. (2000). Sistemas terapéuticos y conflictos culturales. En J. Villalba (comp.), Medicina tradicional en México (pp. 55-78). México: Instituto Nacional de Enfermedades Respiratorias.

Babbie, E. (1999). Fundamentos de la investigación social. México: International Thomson Editores.

Balam Pereira, G. (1991). Cosmogonía y uso actual de las plantas medicinales de Yucatán. Mérida: Universidad Autónoma de Yucatán.

Bermúdez, A. y Velásquez, D. (2002). Etnobotánica médica de una comunidad campesina del estado Trujillo, Venezuela: un estudio preliminar usando técnicas cuantitativas. Revista de la Facultad de Farmacia, 44. Recuperado de http://www.saber.ula.ve/bitstream/123456789/23797/1/ articulo44_1.pdf.

Conti, L. (1972). Estructura social y medicina. Medicina y sociedad. Confrontación. Barcelona: Fontanella.

Flores, J. y Espejel, I. (1994). Etnoflora yucatanense. Tipos de vegetación de la Península de Yucatán, fascículo 3. Mérida: Universidad Autónoma de Yucatán.

García, H., Sierra, A. y Balam, G. (1996). Medicina maya tradicional. Confrontación con el sistema conceptual chino. México: Educación, Cultura y Ecología.

Gubler, R. (1996). El papel del curandero y la medicina tradicional en Yucatán. Alteridades 6(12), 11-18. Recuperado de http://www.uam-antropologia.info/alteridades/alt12-2-gubler.pdf. 
Güemez, M. (1992). Curanderismo rural en Yucatán, persistencia y funcionalidad. Revista de la Universidad Autónoma de Yucatán, 7(182), 37-45.

Hernández, F. (2000). Breve historia de los medicamentos. En J. Villalba (comp.), Medicina tradicional en México (pp.103-123). México: Instituto Nacional de Enfermedades Respiratorias.

INEGI (Instituto Nacional de Estadística y Geografía). (2007). Anuario Estadístico Yucatán, ts. I y II. México: Gobierno del Estado de Yucatán.

König, S. (2011). La medicina indígena: un sistema de salud. Tukari, 3(16), 4-5. $\mathrm{Ku}, \mathrm{A}$. (1992). Medicina indígena y medicina moderna. El cambio en Maní, Yucatán. Revista de la Universidad Autónoma de Yucatán, 7(182), 37-45. Lagarriga, I. (2000). Medicina indígena en México. Conceptos, actitudes y valores de sus seguidores. En J. Villalba (comp.), Medicina tradicional en México (pp. 17-54). México: Instituto Nacional de Enfermedades Respiratorias.

Lozoya, X. (1984). Bibliografía básica sobre herbolaria medicinal de México. México: Secretaría de Desarrollo Urbano y Ecología (Sedue).

May, N. (2007). Evaluación de los recursos maderables y estado de conservación de las selvas del municipio de Tzucacab, Yucatán (Tesis de maestría). Facultad de Medicina Veterinaria y Zootecnia-Universidad Autónoma de Yucatán, Mérida.

Narváez, J. (2001). Estudio etnobotánico de plantas medicinales de la ciudad de Tizimín (Tesis de licenciatura). Facultad de Medicina Veterinaria y Zootecnia-Universidad Autónoma de Yucatán, Mérida.

Polanco, N. (2004). Conocimiento, uso y manejo de plantas medicinales en el poblado de Hocabá, Yucatán, México (Tesis de licenciatura). Facultad de Medicina Veterinaria y Zootecnia-Universidad Autónoma de Yucatán, Mérida.

Segovia, A. (2001). La cacería de subsistencia en Tzucacab, Yucatán, México (Tesis de maestría). Facultad de Medicina Veterinaria y Zootecnia-Universidad Autónoma de Yucatán, Mérida.

Toledo, V., Barrera-Bassols, N. y García-Frapolli, E. (2008). La resiliencia entre los mayas yucatecos: una aproximación etnoecológica. En V. Toledo (ed.), Etnoecología de los mayas yucatecos (pp 63-83). México: Jitanjáfora. 
Viesca, C. (1976). La herbolaria en el México prehispánico. En X. Lozoya (ed.), Estado actual del conocimiento en plantas medicinales mexicanas (pp.11-26). México: Instituto Mexicano para el Estudio de las Plantas Medicinales.

Yam, M., Quiñones, M. y Pérez, J. (1992). La medicina indígena entre los henequeneros y maiceros yucatecos. Mérida: Dirección General de Culturas Populares.

Zamora, P. (2007). Caracterización de la vegetación y su manejo en el municipio de Tzucacab, Yucatán (Tesis de maestría). Facultad de Medicina Veterinaria y Zootecnia-Universidad Autónoma de Yucatán, Mérida. 
